\title{
Trivial Pursuit
}

Abécédaire d'identité pop-folk à l'usage des linguistes, suivant l'usage profane et savant

Guy Achard-Bayle et Amélie Cure

\section{(2) OpenEdition}

1 Journals

Édition électronique

URL : http://journals.openedition.org/pratiques/1178

DOI : 10.4000/pratiques. 1178

ISSN : 2425-2042

Éditeur

Centre de recherche sur les médiations (CREM)

Édition imprimée

Date de publication : 15 décembre 2008

Pagination : 29-57

Référence électronique

Guy Achard-Bayle et Amélie Cure, «Trivial Pursuit », Pratiques [En ligne], 139-140 | 2008, mis en ligne le 15 décembre 2008, consulté le 08 mars 2021. URL : http://journals.openedition.org/pratiques/1178 ; DOI : https://doi.org/10.4000/pratiques. 1178

(c) Tous droits réservés 


\title{
Trivial Pursuit
}

Abécédaire d'identité pop-folk à l'usage des linguistes, suivant l'usage profane et savant

\author{
Guy Achard-Bayle
}

Université Paul Verlaine, Metz, \& CELTED

Amélie Cure

ATILF, pour la troisième entrée

«L'Armana prouvençau, bèn-vengu di païsan, gousta pèr li patrioto, estima pèr li letru, recerca pèr lis artisto...»

Frederi Mistral, Memòri e raconte

\section{Introduction.}

«[Pichoto] publicacioun, souto formo d'armana ». Petite «prose d'almanach » (I) : d'Amélie à Mistral...

Je voudrais donner à ma contribution, c'est à dire à la «pierre » que je voudrais apporter à la réflexion - et aux interrogations - sur la linguistique populaire, une forme « populaire».

Certes, cet adjectif est sujet à caution, tant il « interpelle » et dérange, de par sa filiation, le monde savant; mais nous sommes précisément ici pour l'interroger ( $\leftrightarrow$ Le linguiste et le peuple).

La forme que j'ai choisie, donc, est celle de l'abécédaire ; mais mon intention est également de rendre hommage, par ce choix d'une forme mêlée et fragmentaire, à la « prose d'almanach »; et plus généralement à toutes sortes de formes érudites - dans le sens que donne par exemple à cet adjectif Amélie Cure (2006) lorsqu'elle se penche sur l'activité lexicographique d'un Godefroy dans la deuxième partie du $\mathrm{XIX}^{\mathrm{e}}$ siècle.

Forme érudite donc savante, mais aussi savante et populaire, dans le sens maintenant que donne à cette coordination - à cette conjonction des points de vue, des talents, des sources et des réceptions -, dans le sens que lui donne donc Mistral à peu près à la même époque : il s'en explique dans Mémoires et récits, où il 
raconte et justifie, autant par l'inspiration (folk) que par l'audience (letru) ${ }^{(1)}$, la naissance de L'Armana provençau (Avignon, chez Roumanille, 1855).

\section{...et de Mistral à Amélie (en hommage)}

Qu'un autre hommage lui soit rendu ici. Il ne fallait pas longtemps pour apprécier les talents d'Amélie Cure, jeune et brillante étudiante de master (2006), puis de doctorat. Un accident de voiture lui a ôté la vie à l'été 2007. Elle avait été la première lauréate du concours aux allocations de recherche de notre École doctorale ; toute jeune doctorante, mais déjà bien avancée et assurée dans ses recherches lexicographiques sur l'ancien-français et dans les bases de données textuelles à l'ATILF, elle était revenue en 2007 dans mon séminaire de sémantique référentielle et textuelle pour y faire un exposé, lumineux et savoureux, sur les métaphores du boucher: tueur de bouc et chirurgien! - autrement dit des origines médiévales à aujourd'hui ; sa perspicacité dans le choix des textes et sa finesse dans leur analyse nous avaient ravis, tant, d'humeur joviale, elle avait su jouer de ce nom de métier devenu une métaphore aux facettes bien nombreuses et inattendues, vu notre habitude à l'employer et à l'entendre presque exclusivement pour des mains... charcutières indélicates.

Amélie Cure avait donc consacré ses premières recherches à la lexicographie de 1'ancien français; elle devait ici reprendre, avec son directeur, ses premiers travaux. Je lui laisse donc la parole de ce qui fut sa première proposition de participation au sommaire; d'autant que sa réflexion sur les dictionnaires trouve toute sa place dans cette « publicacioun souto formo d'armana »...

\section{« Le dictionnaire non ou préscientifique et la linguistique populaire » (A. Cure)}

Le dictionnaire non scientifique ou préscientifique n'est pas un objet linguistique spontané : il participe d'un savoir, d'une organisation, d'une méthode. Il ne semble donc pas s'inscrire dans la linguistique populaire. Pourtant jusqu'à la fin du XIX ${ }^{\mathrm{e}}$ siècle, ce type de dictionnaires est l'œuvre d'érudits. À cette époque, l'érudit est considéré par l'institution comme un amateur. Sa démarche, contrairement à celle du spécialiste, est plus « expérimentale », moins scientifique et son savoir, plus empirique.

On peut alors se demander quelle est la part qu'occupe la linguistique populaire dans ces ouvrages et où ils se situent dans cette perspective.

Dans cette étude, je me limiterai à l'examen de deux dictionnaires de l'ancienne langue : celui de La Curne de Sainte-Palaye (1875-1882) et celui de Frédéric Godefroy (1880-1902).

La question des rapports existants entre dictionnaires non ou pré-scientifiques et la linguistique populaire pourra être abordée sous des angles différents. Ainsi on relèvera, tout d'abord, les marques de discours spontané au sein même des articles de ces dictionnaires. On pourra également mettre en lumière le purisme dont fait preuve l'auteur, en ne s'intéressant qu'au « beau langage », par exemple. On se penchera ensuite sur la place de ces diction naires dans l'institution : sont-ils

(1) Il y a pour Rouquette ([1963] 1968: 86), dans le romantisme, puis le néo- ou le postromantisme, qui accompagne à partir du milieu du XIX ${ }^{\mathrm{e}}$ siècle, cette redécouverte d'un peuple et la renaissance de sa langue, une forme de «sentimentalisme et d'ouvriérisme » qui « lui donne l'audience des foules ». 
profanes au sens étymologique, c'est-à-dire "hors du temple » du savoir institutionnel? Leur reconnaît-on une vraie valeur ou sont-ils uniquement sujets aux critiques ? Enfin, une dernière approche consistera à s'interroger sur le continuum entre ces dictionnaires et leurs successeurs scientifiques du $\mathrm{XX}^{\mathrm{e}}$ siècle : sont-ils exploités (et si oui de quelle façon) ou, au contraire, complètement délaissés ?

\section{Petite « prose d'almanach» (II) : principes et contenus}

Ma proposition au sommaire de ce numéro (畞 Principes (savants) d'identité «triviale ») est de réfléchir aux diverses manières dont l'identité est construite en théorie, en des théories plus ou moins «triviales », et de voir notamment à quels besoins linguistiques elle est utile - ou utilisée. Si ma contribution prend la forme de cette " prose d'almanach », c'est en hommage à un type d'écriture, comme je l'ai expliqué et justifié plus haut; mais par précaution, quantitative autant que qualitative, n'étant pas familier du genre, j'ai précisé que ma « prose » serait «petite»; je veux dire par là que l'inventaire ou l'abécédaire (je préfère utiliser ce terme, parce qu'il conjugue l'entreprise et l'organisation encyclopédiques, à la modestie : trivial pursuit) que je proposerai sera forcément partiel, réduit, voire désordonné (en tant qu'alphabet)...

C'est que l'écriture encyclopédique est à la fois plus souple et plus contrainte : elle permet de nombreux renvois et autorise de la sorte une lecture "à sauts et à gambades " suivant le principe ou les allures de Montaigne (cf. ici-même Schmale) ; inversement, elle rend une lecture linéaire et une progression argumentative plus aléatoires : je propose ainsi de mêler les deux démarches, ou les deux allures, en grisant les marqueurs de l'ordre et de la progression...

\section{Principes (savants) d'identité « triviale » (2)}

«C'était ma chambre, et pourtant ce n'était pas la mienne; le sentiment navrant de ma totale impuissance à concilier son apparence et son identité m'accablait et étouffait ma raison. » (Fitz O'Brien, La chambre perdue)

L'idée de traiter la question de l'identité autrement qu'en termes logico-métaphysiques n'est ni nouvelle (Protagoras est sans doute le premier iconoclaste de cette idéalité), ni propre à telle ou telle discipline des sciences humaines autre que l'ontologie ou la philosophie du langage ${ }^{(3)}$. Ceci dit les modèles que ces dernières perpétuent ( $c f$. le «néo-aristotélisme» de Ferret, 1996) restent dominants en tout cas dans notre champ d'investigation linguistique ( $c f$. Reboul, 1993 et 1997) ${ }^{(4)}$.

Traiter l'identité, toutefois, demande des "échanges interdisciplinaires » (Lévi-Strauss, $1987: 9)$ :

(2) Cette approche de l'identité a fait l'objet d'une communication au colloque de Tours des 29 et 30 novembre 2007 : Construction d'identité et processus d'identification (cf. AchardBayle, 2007 à par.).

(3) Voir l'anthropologie : Lévi-Strauss ([1962] 1990) sur les noms d'espèce ; sur l'anthroponymie : Zonabend ([1977] 1987), Bromberger (1982). Pour une approche logico-poétique : Molino (1982).

(4) Voir l'anthropologie ( $c f$. note 3 ). On pourrait en dire autant de l'identité à travers le temps, du temps et de la continuité, de l'identité narrative (Ferret, 1993 ; de Ricœur [1983-1985/ 1991], 1990, 1991 ; Elias, [1984 / 1996]). 
« Car le thème de l'identité se situe non pas seulement à un carrefour, mais à plusieurs. Il intéresse pratiquement toutes les disciplines » (ibid.).

Pour ma part (1997, 2001), je me suis efforcé de présenter d'autres modèles, certes de moindre impact. Un exemple : " l'identité artificialiste » de Rosset ([1984] 1993), qui prend le contrepied de la tradition idéaliste-essentialiste, ne trouve aucun écho face aux hypothèses de métaphysique-fiction développées par les philosophes d'inspiration plus ou moins analytique (Wiggins, 1980 ; Kripke, [1980/1982]; Engel, 1989 ; Engel \& Nef, 1988 ; Ferret, 1993 et 1996) ${ }^{(5)}$. On peut l'expliquer par le fait que le traitement logique ou logico-sémantique des problèmes de l'identité sous la forme de puzzling cases (㖤 Puzzling cases) convient mieux à notre exigence d'abstraction garantie de scientificité ? ${ }^{(6)}$

Bref, on peut se sentir à l'étroit dans ce cadre de modèles dominants. Mais il faut dire que si je m'en suis quelquefois « échappé », c'était plus pour traiter de cas atypiques (Achard-Bayle, 2006 à par.), ou en marge de ma réflexion sur les référents évolutifs (Achard-Bayle, 1996), que pour trouver des modèles de remplacement. L'avantage de l'expérience, toutefois, est qu'elle encourage à renouveler l'école buissonnière, et sinon à chercher d'autres modèles encore, du moins à tenter l'aventure sur d'autres terrains... Or la fréquentation des textes, qui sont un excellent reflet quand bien même des miroirs grossissants / déformants de notre réalité complexe, multiplie les difficultés à résoudre (吗 Cas et traitements littéraires de métaphysique fiction). Il s'agit donc bien de trouver non pas une, mais d'autres voies de résolution.

\section{L'Identité « triviale » (II)}

L'identité dite " triviale » est en fait une construction proprement savante. Pourquoi « triviale », alors ? Parce qu'elle repose sur (expose) une relation d'identité, d'égalité ou d'équivalence, dite " réflexive »:

$$
\forall x(x=x)
$$

[Quel que soit $x, x$ est (nécessairement) égal à lui-même]

D'où :

$$
\begin{gathered}
\text { Napoléon }=\text { Napoléon (identité triviale) } \\
\text { Napoléon }=\text { Vercingétorix (égalité fausse) } \\
\text { Napoléon }=\text { Bonaparte (identité ni triviale ni fausse) } \\
7+5=12 \text { (identité vraie mais non triviale) }
\end{gathered}
$$

La troisième relation est celle qui intéresse(ra) le plus les linguistes, notamment à partir de ce qu'en dira et fera Frege ([1892] 1971) notamment à partir de ou grâce à la différence qu'il instaure entre Sens et dénotation.

\section{L'identité relative : relativité de l'identité (I)}

L'autre apport fondamental de Frege est sa définition de 1'identité qu'on peut déjà dire relative; c'est à dire relative à une identification, donc à un point de vue (de locuteur) : c'est le fameux exemple de Vénus qui peut être (dite / désignée par)

(5) Voir encore Rank, Bettelheim et Todorov pour des interprétations de 1'identité par la psychanalyse, la folks psychology, ou la poétique du fantastique.

(6) Je ne nie pas que l'interprétation des avatars pose bien des questions de logique (formelle ou naturelle); j'essaie d'en faire la démonstration, après Charolles (1997), dans AchardBayle (1997). 
L'étoile du matin ou L'étoile du soir - en fonction notamment des temps et des lieux, autrement dit des contextes d'observation et d'interlocution... Soit, suivant le principe d'identité ni triviale ni fausse, $a=b$.

À ce propos Frege (op. cit. : 102) fait remarquer que les propositions $a=a$ et $a=b$ n'ont pas « la même valeur de connaissance » :

La première pose la relation a priori qu'un objet entretient nécessairement avec lui-même ${ }^{(7)}$, et que nous retrouvons chez Ricœur (par ex. 1990) sous la forme de l'ipséité.

- La seconde suppose une relation, et pas d'équivalence a priori: on peut en effet découvrir, grâce à la recherche scientifique, que deux référents que l'on considérait et appelait diversement (par exemple l'étoile du matin et l'étoile du soir) sont en fait une seule et même entité (Vénus).

Geach (1972) reprendra plus tard cette thèse de la relativité de l'identité, et sous une forme tout aussi - explicitement - énonciative qu'épistémique (heuristique et épistémologique) :

« Je soutiens que l'identité est relative. Quand quelqu'un dit "x est identique à y", ceci, selon moi, est une expression incomplète ; c'est une abréviation pour " $\mathrm{x}$ est le même A que y" où "A" représente un certain nom de chose nombrable [count noun] qui doit être compris à partir du contexte d'énonciation. » (op. cit. : 238.)

Selon cette thèse, la propriété identifiante A est un prédicat sortal, i.e. qui indique quelle sorte d'objet sont $\mathrm{x}$ et $\mathrm{y}^{(8)}$. Ainsi, il n'y a pas d'identité sans critère sortal d'identité, ce qui fait que le célèbre exemple de Frege peut être revu comme suit :

"Ainsi dire que l'étoile du soir est l'étoile du matin, ce n'est pas dire que deux objets ainsi nommés sont identiques, mais plutôt que ce sont la même planète, ou le même corps céleste. » (Geach, ibid.) ${ }^{(9)}$

Pour autant, si deux objets sont le même F (ou ont le même F pour prédicat commun : $a=F b$ ), ils ne sont pas (n'ont pas pour prédicat commun) le même G, le même $\mathrm{H}$, etc. - contrairement à ce qui est affirmé par la première des « Lois de Leibniz 》(烸 Identité et «bon sens »).

\section{L'Identité triviale « revue à la baisse »}

Ce n'est donc qu'un paradoxe apparent : l'identité (dite) «triviale » peut être ainsì « revue à la baisse », sous cette / une forme relative ou relativisée. Sortie des calculs ou des propositions de la logique formelle, elle est censée répondre aux contraintes ou aux contextes de la logique dite «naturelle», y compris en faisant face à des énoncés a priori contradictoires :

«C'était ma chambre, et pourtant ce n'était pas la mienne ; le sentiment navrant de ma totale impuissance à concilier son apparence et son identité m'accablait et étouffait ma raison. » (Fitz O’Brien, La chambre perdue) ${ }^{(10)}$

(7) Ainsi, précise Engel (1989: 245), « l'une des propriétés de $a$ est que nécessairement il est identique à $a »$, ou encore que «l'identité avec soi-même [est] une propriété nécessaire de cet objet» (ch. IX, section 6, "Identité et nécessité »).

(8) La notion de prédicat sortal vient de : Essay on Human Understanding de Locke (1690).

(9) Pour Engel (1982: 433), il n'y a pas de la sorte d'identité abstraite.

(10) Trad. Papy (éd. citée : 786). 
Cette identité « revue à la baisse», ou relativisée, permet ( $c f$. Engel, $1989: 239)$ en outre de résoudre quelques paradoxes d'identité (咹 Puzzling cases) : de la sorte, le docteur Jekyll sera le même homme que Mr Hyde, mais pas la même personne, ou le même caractère; on pourra se baigner deux fois dans le même fleuve, et pourtant pas dans la même eau (嗐 Identité dans le temps).

\section{L'Identité dans le temps : relativité de l'identité (II) ${ }^{(11)}$}

Pour résoudre ledit paradoxe du fleuve d'Héraclite :

«Dans les mêmes fleuves / nous entrons et nous n'entrons pas. / Nous sommes et nous ne sommes pas. » ${ }^{(12)}$

Quine ([1960] 1978, 1961) propose une théorie de la relativité de l'identité qu'on pourrait dire « einsteinienne » dans la mesure où elle s'inscrit dans l'espacetemps ; mais cette conception de l'identité relative, qui intègre la quatrième dimension temporelle aux trois dimensions spatiales, est aussi une conception de bons sens, dans la mesure notamment où cette conception - ou idée - de l'identité résulte de l'expérience que nous faisons, en nous-mêmes, de nous-mêmes et des autres - et par autres, il s'agit de viser non seulement les autres que moi, les personnes dotées de prédicats physiques et psychiques et de la capacité d'autoréflexion, mais également toutes sortes de corps, entités, particuliers ou individus matériels ( $c f$. Ricœur, 1990 et 1991 ; il s'inspire entre autres de Strawson, [1959] 1973 : Individus \& particuliers II).

Revenons à Quine ; pour lui, il faut appréhender le temps dans les mêmes termes étendus que l'espace, donc considérer les objets de manière quadridimensionnelle dans l'espace-temps ${ }^{(13)}$. En conséquence les objets physiques ne seront pas distingués des processus, et le fleuve sera des phases de fleuve, une entité dans ou à travers le temps ${ }^{(14)}$. Du point de vue linguistique, on voit ainsi les catégories de discours - elles-mêmes inspirées des catégories de l'ontologie ou de la métaphysique aristotélicienne ${ }^{(15)}$ - se rapprocher voire se confondre : en l'occurrence substantifs et procès.

\section{L'Identité narrative : l'expérience quotidienne de la continuité et du changement ${ }^{(16)}$}

L'importance du temps dans la détermination de l'identité se retrouve à la première place chez Locke (1690: ch. XXVII); en effet, c'est en comparant une chose avec elle-même dans des temps différents que nous nous formons les idées du même et de l'autre. Ricœur (notamment dans ses ouvrages cités, de 1983 à 1990) poursuivra ce raisonnement, fondé sur l'expérience ordinaire, comme on l'a dit, pour tenter de résoudre l'aporie qui résulte de la conjugaison ou de la juxtaposition et du sentiment (surtout intime) de l'identité (ou de la continuité

(11) Je reprends et adapte ici Achard-Bayle (2001: 46-47).

(12) Fragments d'Héraclite, B, XLIX, trad. Dumont (1991 : 77).

(13) Quine ([1960] 1978, section «Le temps » : 243 sq.). Quine parle ailleurs de « concept d'objet large » (1961:68).

(14) Quine ([1960] 1978: 173 sq.) et (1961: 65 sq.).

(15) Voir Arnauld (1660, éd. numérisée, Paris, BNF, Gallica, « Seconde partie de la grammaire générale », ch. II « Des Noms » et suivants : 59 et sq.) et Arnauld \& Pierre Nicole ([1662] 1970 , ch. III et suivants : $77 \mathrm{sq}$.).

(16) Je reprends et adapte de nouveau Achard-Bayle (2001 : 56-62). 
dans le temps), et du constat du ou des changements (sur soi, sur autrui : autre face de l'identité dans le temps).

À l'échelle de soi (de soi-même comme un autre), l'identité sera narrative : autre emprunt à Aristote (Poétique), l'identité est conçue ici comme un récit - avant d'être un récit - en ce qu'elle permet de confondre (吼 fin de Relativité de l'identité II) ou plus exactement ici de configurer l'identité - ou la mêmeté ${ }^{(17)}-$ et la diversité ; la configuration est ainsi (comme) une « mise en intrigue », elle permet d'intégrer à la permanence (ou au sentiment de celle-ci) dans le temps ce qui en est, apparemment, le contraire : la variabilité ; ainsi, comme dans la grammaire du récit aristotélicienne, il faut atteindre à un équilibre entre concordance (l'agencement des faits suivant un principe d'ordre) et la discordance (les renversements de la fortune).

\section{Identité relative \& identité dans le temps (Implications linguistiques : identité sortale, caractérisation, catégorisation et désignation}

Les théories relativistes de l'identité (吗 Relativité de l'identité I \& II) ont pour corrélat épistémique et discursif de (faire se) poser la question des critères d'appartenance sortale : il s'agit en effet de savoir ce qui permet de définir une espèce pour laquelle seront vrais ou non tels ou tels jugements d'identité ; et par conséquent, pour laquelle seront vraies telles ou telles propositions exprimant, notamment par des désignations catégorisantes, lesdits jugements.

Engel (1989) pense comme les auteurs cités précédemment ( 1 Geach et Quine ou Locke, Strawson et Ricœur in Relativité de I'identité I \& II) que la continuité temporelle et la mémoire vont de pair pour établir " un critère sortal constant» (Engel, op. cit. : 244) - ou suivant Wiggins (op. cit.) la « dépendance sortale », i.e. l'appartenance à une espèce :

«Un prédicat sortal prescrit des conditions de persistance qu'un objet ne peut pas ne pas exemplifier à tout moment de son histoire, sous peine de cesser d'être cet objet. » (Engel, op. cit. : 238)

La prise en compte de la continuité temporelle oriente ainsi vers une autre identité : l'identité sortale; en effet, non seulement la vérité d'une expression du type $a=b$ dépendra de la condition relative que $a$ est le même quelque chose que $b$, mais aussi du fait que des objets seront ré-identifiables, trivialement ou communément, ou encore quotidiennement, comme les mêmes à travers le temps. Ce principe de double similarité, selon Quine ([1969] 1977, § «Espèces naturelles » : 133-139), garantit l'apprentissage et l'usage des mots : il fait d'un côté qu'un terme est applicable à tous les échantillons d'une même espèce, de l'autre, que ses occurrences passées sont mises en rapport avec les présentes.

\section{Identité diachronique \& Identité spécifique. Catégorisation \& désignation (II)}

Ferret (1996) pose également la question du paradoxe de l'identité et du changement :

« [O]u bien le particulier qui change demeure un et le même et alors il n'a pas changé $[\ldots]$ ou bien le particulier a vraiment changé et alors il n'est plus un et le même. » (op. cit. : 21)

(17) Autrement dit le concept de sameness repris de Locke (op. cit.). 
Mais en philosophe analytique, il s'intéresse aussi à la question sous l'angle des procédures qui permettent l'individuation donc la désignation :

«Si identifier un particulier avec lui-même est une trivialité et si identifier deux particuliers entre eux est une absurdité ${ }^{(18)}$, un examen des conditions d'identification et de réidentification d'un seul particulier avec lui-même à deux moments distincts de sa carrière s'inscrit au cœur des grands problèmes traditionnels de persistance, de changement et d'individuation des particuliers. » (op. cit. : 10)

Ferret (1996) commence par faire la « grammaire du même ». En effet,

« la thèse selon laquelle le changement détruit ipso facto l'identité du particulier qui s'en trouve affecté repose sur une confusion entre deux sens de "même": le même dénotant l'identité numérique, et le même dénotant l'identité qualitative. » (op. cit. : 27) ${ }^{(19)}$

Ainsi s'achemine-t-il vers une identité diachronique qui permet de concilier identité et changement; et si « un particulier demeure un et le même », c'est qu' «il peut être recouvert par au moins un même concept sortal tout au long de sa carrière » $(\text { op } \text {. cit. : } 37)^{(20)}$; par exemple être un homme (vs être un philosophe) en ce qui concerne Kant. Ainsi, " la sorte à laquelle appartient un particulier constitue la frontière infranchissable par le même » (ibid.), et l'énoncé Kant est un homme n'a de valeur de vérité que jusqu'au point limite où Kant devient alligator (

\section{Identité sortale (II) : le phénotype et les folk ontologies}

Il y a des changements radicaux (plus ou moins naturels donc factuels et inversement contrefactuels) qui provoquent un changement d'espèce (en termes logico-philosophiques : «le passage d'une sorte à une autre », Ferret, $1996: 38^{(21)}$ ) au titre desquels les métamorphoses...

Mais qu'est-ce qui permet de déterminer ce qui fonde la sorte d'un particulier? N'en déplaise aux nominalistes,

«La question qui se pose est celle de la réalité sortale extralinguistique. » (Ferret, $1996: 45)$

Mais que les nominalistes ou les « conventionnalistes » se rassurent :

«Si le découpage humain du monde s'avère exclusivement symptomal, alors les critères d'identité spécifique se ramèneront à des conventions, communément ou non acceptées. » (ibid.)

Il y a donc diverses façons - ou conventions - de déterminer la sorte : entre autres (toujours suivant Ferret, 1996) la macrostructure (1'apparence ou

(18) Cf. Wittgenstein ( Identité et «bon sens »).

(19) Dans son ouvrage antérieur, Ferret (1993) distinguait un même accidentel et un même essentiel ou nécessaire. C'est ainsi que le fameux morceau de cire de Descartes (Méditations, II) est et n'est pas le même, suivant qu' on le considère numériquement ou qualitativement. Du point de vue numérique, l'identité équivaut non à la ressemblance ou à la similitude, mais à « une coïncidence complète de substance» (Ferret, op. cit. 1996:29).

(20) Ou encore : «Pour appartenir à la carrière d'un seul et même particulier, deux occurrences de particulier doivent au moins être sortalement identiques » (op. cit. : 84).

(21) Suivant Aristote, ce sont des changements par génération ou corruption; les changements qualitatifs relèvent, eux, de l'altération (De la génération et de la corruption, 319, b, 7-18). 
phénotype), la microstructure (la constitution interne), enfin l'attribution fonctionnelle.

Mais suivant la tradition, les Anciens, on aurait pu commencer par dire : la " génération », autrement dit, la filiation donc l'inscription génétique dans l'espèce. Toutefois, comme le remarquait Locke $\left(1690\right.$, III, VI) ${ }^{(22)}$, se pose le cas des hybrides : le mulet par exemple, fruit d'un croisement de deux espèces, le cheval et l'âne...

Le phénotype («ou la description phénoménologique d'un particulier », Ferret, op. cit. : 48) n'est-il pas un critère plus satisfaisant? La réponse est assurément affirmative pour une philosophie (proche) du sens commun :

«[B]ien des gens déterminent les espèces plutôt par leur forme extérieure que par leur naissance. » (Locke, ibid.)

«C'est la forme extérieure, chez les êtres produits par la nature, qui permet de reconnaître leur espèce. » (Thomas d'Aquin, Somme théologique, III, 74, III, cité par Ferret $1996: 48$, note 3)

À suivre.../... (吼 Les Apparences trompeuses)

\title{
Identité / Identification \& « Réalité des espèces " (Verbatim : petit plaidoyer pour la «Réalité des espèces »)
}

\begin{abstract}
« Dans la jungle, où personne n'a jamais mis les pieds, les lions sont des lions, et les girafes sont des girafes. En revanche, c'est nous qui décidons, notamment par l'utilisation qu'on en fait, que "ceci" est un "bateau" ou que "ceci" est une "chaise". Les organismes sont gouvernés par une essence réelle, intrinsèque, indépendante de l'esprit et des idéologies. Les artefacts ne sont conditionnés que par une stipulation nominale extrinsèque. Enfin, ce n'est pas parce que notre classement actuel des espèces n'est pas fidèle à la réalité que cette réalité des espèces n'existe pas in concreto. Une chose est de dire que c'est nous qui découpons le monde de telle et telle manière (ce que personne ne songe à nier), une autre est de dire que le monde lui-même se prête à être découpé de telle ou telle manière.

Sommes-nous donc comme un aveugle qui essayerait de classer les couleurs ou pouvons-nous espérer réussir à faire coïncider notre découpage du monde avec les structures du monde lui-même ${ }^{(23)}$ ? Pour employer une métaphore célèbre, avons-nous [sic] ou pouvons-nous espérer découper le poulet-monde dialectiquement au sens de Platon, c'est à dire en en suivant précisément les articulations même du poule-monde, ou bien sommes-nous contraints de le débiter en tranches anonymes et fades ?» (Ferret, $1996: 46-47)$
\end{abstract}

Pour prolonger le débat : $c f$. la distinction que fait Searle ([1996] 1998) entre faits bruts et faits institutionnels. Sur les « conventions communément ou non acceptées », cf. Sperber (1996) et sa contagion des idées. Mon plaidoyer personnel en faveur d'un réalisme minimal / -iste: Achard-Bayle (2008 : ch. III, 19-25). Ici même : Larsson et son «Bon sens commun ». Vignaux (1999) et son Démon du classement.

(22) Ferret (1996) cite Locke dans l'éd. fr. de 1972 (en l'occurrence la page 26).

(23) Note de Ferret : «Cf. Leibniz, Nouveaux Essais [sur l'entendement humain], III, VI, [éd. Paris, Flammarion] G.-F., pp. 263-264». 


\section{Identité spécifique $\&$ "préjugés pragmatiques" (Verbatim II : le «découpage préthéorique» du monde)}

«Il est navrant de constater que de nombreuses théories de l'identité spécifique considèrent que le découpage humain en espèces ou en sortes ne dépend que de nos intérêts particuliers, notamment économiques. Le découpage préthéorique était, certes, conditionné par des raisons d'ordre pragmatique. Les premiers herbiers, notamment, répertoriaient les plantes en fonction de leurs vertus médicinales ou de leur comestibilité. En revanche, on ne voit pas pourquoi les espèces actuellement définies seraient encore conditionnées par des raisons de ce genre. Les critères d'identité ne dépendent pas de notre bon vouloir. Par exemple, "être un fruit" ne dépend ni de nos croyances, ni de nos intérêts, ni de nos usages personnels [...]. Le fait que la grande majorité des consommateurs considère que les tomates sont des légumes ne transforme pas ces particuliers en légumes. Les tomates sont des fruits et le resteraient même si $100 \%$ des hommes étaient prêts à soutenir le contraire. Être un fruit correspond à une définition transculturelle univoque, précisément celle que l'on rencontre dans le dictionnaire. » (Ferret, $1996: 47$ )

Cette longue citation appelle quelques commentaires : l'identité est finalement nominale dans la mesure où elle est en dernier ressort consignée, i.e. définie dans et certifiée conforme par le dictionnaire, en tant que lieu d'inscription d'une communauté de sens. Mais l'exemple de la tomate montre bien que cette communauté de sens institutionnalisé ou savant (que Ferret appelle univocité transculturelle) ne va pas forcément de pair, bien au contraire, avec le (bon) sens commun, ordinaire, qui se définit lui comme un rassemblement de voix au sein d'une communauté de sens établie par intersubjectivité (cf. Kleiber 1997, 1999, 2001, «Le bon sens commun »).

\section{«La division du travail linguistique » (Verbatim III)}

L'écart entre le sens établi et le (bon) sens commun peut être expliqué par ce que Putnam a appelé la « division du travail linguistique » :

« Every linguistic community exemplifies the sort of division of linguistic labor as : possesses at least some terms whose associated "criteria" are known only to a subset of the speakers who acquire the terms, and whose use by the other speakers depends upon a structured cooperation between them and the speakers in the relevant subsets. » (Putnam, $1975: 228)$

Ceci revient à dire que la compétence sémantique n'est pas répartie, ou distribuée de manière égale ou homogène dans une communauté linguistique, autrement dit également entre tous les membres de cette communauté. (Inversement ou complémentairement : Larsson et « Le bon sens commun».)

\section{Les Apparences trompeuses... et le retour (le recours savant) aux essences et/ou aux noms}

Pour Putnam (op. cit.) - qui l'a montré, il faut le noter, à propos des noms d'espèces -, aucune propriété phénotypique n'est vraiment nécessaire à la description d'un particulier : un citron vert est un citron même s il ne mûrit jamais, un tigre sans rayures appartient quand même à l'espèce...

Pour la philosophie (contemporaine), la constitution interne (ou microstruc- 
ture) paraît un critère plus sûr dans la mesure, notamment, où c'est à la science qu'il revient de la déterminer. Ceci dit, outre qu'il y a un lien entre la microstructure et la morphologie et donc la phénotypie d'un particulier, on n'a pas attendu, pour désigner les espèces, les substances et leurs spécimens, que les savants en aient donné la description scientifique ( $c f . \mathrm{H}_{2} \mathrm{O}$ pour l'eau).

C'est que, suivant Kripke ([1980] 1982), les noms d'espèces naturelles et de substance, comme les noms propres, qui sont pour lui des noms rigides, ne sont pas des conjonctions de propriétés (vs Putnam, 1975, qui plaide pour cette « conjonction spécifique ») : leur signification est fournie par un acte de baptême, tel que, par exemple, « un jour, cette charmante créature est désignée comme chat » (Ferret, $1996: 67)^{(24)}$. Et si ces noms sont rigides, c'est que leurs porteurs ne sont pas ou plus exactement ne sont plus dès lors censés en changer ${ }^{(25)}$.

Le résultat de cet acte de langage est qu'il détermine un prototype; et, de même qu'il est tout-à-fait possible d'assimiler à ce dernier des spécimens présentant d'éventuelles différences phénotypiques, on lui associera sans peine, une fois découverte, la description scientifique des espèces ou substances. L'identification sortale, qui détermine l'appartenance à l'espèce, est donc (aussi, surtout?) affaire de désignation... ${ }^{(26)}$

\section{Apparences trompeuses (II) : de l'empirisme et au nominalisme - qualités premières et secondes}

Les théories relativistes de l'identité sont, comme dit Quine ([1969] 1977:63), des « doctrines relationnelles ». Elles conduisent ainsi à considérer les identités non plus en soi mais en fonction de coordonnées. Autrement dit, et on l'a dit, contextuellement.

Les partisans du réalisme posent alors la question : les concepts et leurs noms, réfèrent-ils, désignent-ils seulement des manières de penser ou des entités indépendantes de nos manières, ou conventions, de penser (Nef, 1991:104) ?

Cette question renvoie au débat, ancien, qui opposait et d'une certaine manière continue d'opposer essentialistes (ou réalistes) et nominalistes (吼 Identité / Identification \& « Réalité des espèces »).

Locke distingue pour sa part essence réelle et essence nominale. Pour lui en effet, l'essence réelle est ce qui concerne la substance et ses qualités premières - ces qualités sont non visibles, comme le sont, par exemple, la structure atomique et moléculaire d'un corps en or. Ces corps ont néanmoins :

«[L]a puissance de produire diverses sensations en nous par le moyen de leurs qualités premières [...] comme les 7 couleurs, les sons, les goûts etc. » ${ }^{(27)}$

(24) «Le concept initial de chat est: "cette espèce de chose", où l'espèce peut être identifiée au moyen d'exemples paradigmatiques » (Kripke, op. cit. : 111).

(25) Pour les changements de noms propres, notamment dans les sociétés traditionnelles, voir les travaux cités de Zonabend ou Bromberger, et Lévi-Strauss. J'y ai moi-même consacré une étude qui porte sur une fiction dans la France rurale du XIX ${ }^{\mathrm{e}}$ siècle (Achard-Bayle, 1996).

(26) Je laisse volontairement de côté les théories linguistiques et psycho- ou anthropo-linguistiques des prototypes, je les suppose davantage connues des linguistes, que les théories onto/logiques : voir en bibliographie Kleiber (1990), Dubois (bibliographie dans sa dernière publication 2008), Wierzbicka (1988: ch. 10, 1996).

(27) Essai... (Livre II, ch. 8 « Autres considération sur les idées simples », § 10 sans titre, trad. Coste, revue par Thurot, Tome II des Euvres philosophiques de Locke, 1821, Paris, Firmin Didot, en ligne sur Google Books : 265 ; téléchargeable). 
Et Locke donne à ces qualités « le nom de qualités secondes »-ou qualités sensibles ; par exemple, jaune, lourd pour le corps en or ${ }^{(28)}$.

Quant à l'essence nominale, elle correspond à ce que nous concevons à partir d'expériences répétées, et - précise Locke qui porte une attention toute particulière à l'activité linguistique qui résulte de notre activité empirique, ou de notre expérience du monde -, à ce que nous disons d'un corps ou de ce corps, après l'avoir expérimenté, ressenti...

Mais Locke, en nominaliste modéré, met en garde contre la confusion qu'on serait amené à faire ou qu' on pourrait faire entre ces deux types d'essence; et donc contre cet

« abus qu'on fait des mots [qui est ou serait de] les mettre à la place des choses qu'ils signifient. » ${ }^{(29)}$

Poussé plus avant, l'empirisme de Locke mène au «fictionnalisme » de Hume pour qui, effectivement, l'identité n'est qu'une illusion ; une illusion de permanence que notre imagination construit en superposant des perceptions successives :

« Je ne m'atteins jamais moi-même à un moment quelconque en dehors d'une perception et ne peux rien observer d'autre que ma perception. » (Hume, Traité de la nature humaine, Livre I, cité par Ricœur, 1990 : 154)

Quine (1961, ch. IV, section 1 «Identity, Ostension, and Hypostasis » : 66-67) considère cette conception de Hume comme «interesting as a psychological conjecture on origins », mais il rejette l'hypothèse d'un "nouvel objet non momentané » («nonmomentary »), identifié ou reconstruit à partir d'événements ponctuels séparés dans le temps; à l'inverse (吗 L'identité dans le temps : relativité de l'identité II), il propose d'une part de mettre en relation identité ou substance et procès, de l'autre de considérer les objets comme « étalés dans le temps » («time-extended »).

\section{Des essences minimales : individus \& particuliers (I)}

Face au risque que fait courir un relativisme extrême, la prudence - qui relève aussi du bon sens ou du sens commun aussi bien chez le linguiste, que le logicien philosophe du langage, particulièrement s'il est analytique, peut-être même le métaphysicien -, conduit à des positions et des propositions essentialistes «minimales ». Ainsi pour Strawson ([1959] 1973 : 37),

«le scepticisme philosophique nous met devant l'alternative soit de vouloir dire quelque chose de différent de ce que nous disons, soit d'être à jamais dans l'incertitude. »

Strawson - contre ce scepticisme - accepte sans condition ( « sans question » ditil) le principe de l'identité des individus suivant la capacité que nous avons de référer à (i.e. identifier et ré-identifier) ceux-ci « dans au moins certains cas d'observation non continue ». Ainsi l'individu est-t-il saisi dans la durée et reconnu en tant que «le même individu ». Il faut néanmoins considérer cette mêmetécomme relevant de deux identités, donc comme révélant chez un individu : son identité numérique, qui le définit comme le «même-un », et son identité qualitative, qui le définit comme le « même-semblable» (Strawson, op. cit. : 34 à 38).

(28) Voir les commentaires d'Engel (1984), Ricœur (1990: $150 s q$.$) et Nef (1991).$

(29) Essai... (Livre III, ch. 10 " de l'abus des mots», § 17 On donne aux mots une signification qu'ils ne peuvent avoir, éd. citée, tome IV, 1823, Paris, Firmin Didot, en ligne sur Google Books : 62 ; téléchargeable). 
Strawson passe ensuite (op. cit. : $41 s q$.) à la caractérisation des particuliers qui peuvent se présenter soit comme des corps matériels (avec des qualités tactiles ${ }^{(30)}$ ) soit comme des états, des processus appliqués à (ou subis par) lesdits corps. Ces distinctions conduisent à définir, isoler et caractériser les « particuliers de base » («basic particulars »), qui doivent être entendus indifféremment comme des personnes, des animaux ou des choses ${ }^{(31)}$.

Si l'on considère maintenant que notre cadre de référence est quadridimensionnel, on voit alors que seuls les corps matériels réussissent à le " remplir »; ce qui veut dire, notamment, qu'ils réussissent à remplir une des conditions de l'identité, à partir du moment où celle-ci est conçue comme « continuité de l'existence dans le temps » (op. cit. : 61).

Si l'on considère ensuite que les corps matériels peuvent être directement identifiés ou ré-identifiés dans ce cadre spatio-temporel, c'est à dire sans qu' on ait besoin de se référer pour cela à d'autres particuliers, alors ces corps matériels fournissent la base de l'identification des particuliers ; autrement dit, ces corps matériels sont des particuliers de base (op. cit. : 60 et 97).

\section{Genèse des langues (notamment des noms concrets) et réalisme « naïf »}

On se retrouve ainsi avec l'idée, depuis longtemps répandue et somme toute triviale, que les noms concrets, référant à des objets matériels ou servant à les identifier, témoignent mieux que les autres noms et mots du réel. Ainsi pour le sensualiste Condillac :

«La langue fut longtemps sans avoir d'autres mots que les noms qu'on avait donnés aux objets sensibles. [...] On distingua ensuite, mais peu à peu, les différentes qualités sensibles des objets, on remarqua les circonstances où ils pouvaient se trouver et l'on fit des mots pour exprimer toutes ces choses : ce furent les adjectifs et les adverbes ; mais on trouva de grandes difficultés à donner des noms aux opérations de l'âme, parce qu'on est naturellement peu propre à réfléchir sur soimême. " ${ }^{(32)}$

Cette théorie de la genèse du lexique des langues est proche des propositions du « réalisme naïf » de Lyons ([1978] 1990 : 74 sq.) qui consiste en la distinction de trois ordres d'entités (et de mots) : les entités du premier ordre, qui rassemblent les objets physiques, perceptibles par tous, s'inscrivent dans l'espace à trois dimensions ; celles du deuxième ordre, les événements, les processus et les états, qui s'appliquent aux premières, «n'existent pas » mais « ont lieu »; les entités abstraites, enfin, échappent au temps comme à l'espace.

\section{Individus \& particuliers (II) : l'identité personnelle et le dualisme « classique»}

Strawson ([1959] 1973 : 97-130) se penche également sur un type de particulier, la personne :

« [cette] entité telle qu'à la fois des prédicats attribuant des états de conscience et des prédicats attribuant des caractéristiques corporelles, une situation physique, etc.,

(30) C'est «1'extension » de Descartes ou la « solidité » de Locke.

(31) Voir aussi les «entités du premier ordre » ou « objets physiques » chez Lyons ([1978] 1990, $2.3: 74$ sq.).

(32) Cette citation, sans référence, est empruntée à Sumpf (1984:115). 
sont également applicables à un seul individu de ce type unique. » (Souligné par 1'auteur, op. cit., ch. 3, section 4 « Nature primitive du concept de personne » : 114)

Suivant la formalisation de Ferret (1993:21), ces prédicats de deux types sont les prédicats- $M$ (comme matériels), et les prédicats- $P$ (comme psychologiques). On se retrouve donc ici avec une définition, double, qui s'inscrit bien dans une longue tradition, dualiste, que Ferret (1993 : 21) fait remonter à Boèce :

"Persona naturae rationalis individua substantia »/ La personne est une substance individuelle de nature rationnelle. ${ }^{(33)}$

Strawson continue également cette tradition en mettant en avant les prédicats psychiques ou mentaux chez les particuliers que sont les personnes. En dernier lieu, ce sont en effet les prédicats- $P$ qui distinguent l'individu ou le particulier personnel des « corps exclusivement matériels », auxquels il est « illégitime » d'appliquer ces prédicats - suivant le terme de Ferret $(1993: 22)^{(34)}$. Dès lors (Ferret, op . cit. : 49) l'identité personnelle sera définie (et l'identité d'une personne en particulier reconnue) suivant deux critères, l'un corporel et l'autre psychologique ; ainsi :

$[\mathrm{U}]$ ne personne $\mathrm{P}$ sera identique à elle-même et / ou ré-identifiée comme la même $\mathrm{P}^{*}$ (dans le temps), et / ou par le fait que $\mathrm{P}$ et $\mathrm{P}^{*}$ auront le même corps, et / ou par le fait qu'il existera « une relation psychologique interne directe» entre $\mathrm{P}$ et $\mathrm{P}^{*}$. (Je m’inspire ici de Ferret, 1993 : 49, ch. III « Le corps, la mémoire et le temps »)

Locke définit aussi la personne dans sa dimension spirituelle, ou plus exactement par (l'activation de) ses facultés intellectuelles :

« [U]n Être pensant et intelligent, capable de raison et de réflexion, (i) qui peut se consulter lui-même comme le-même, (ii) comme une même chose qui pense en différents temps et en différents lieux. "(Essai, II, ch. XXVII, Ce que c'est qu'Identité, \& Diversité / On Identity \& Diversity, § 9 En quoi consiste l'Identité personnelle/ Personal identity; j'ajoute les parenthèses i et ii) ${ }^{(35)}$

Mais il donne à cette définition, si l'on peut dire, davantage d'ampleur et d'actualité, en mettant l'accent sur les propriétés (i) de réflexion et (ii) de continuité qui, à partir de lui, caractériseront l'identité personnelle. Ces propriétés vont donc au-delà du dualisme classique ou traditionnel et annoncent la distinction fondamentale que fait Ricœur (1990) entre mêmeté ${ }^{(36)}$ et ipséité.

(33) Dualité de la personne et dualisme de sa conception sont naturellement en rapport avec la problématique des métamorphoses que les philosophes utilisent comme puzzling cases (吼 Puzzling cases).

(34) Dans le même ouvrage, Ferret cherche néanmoins de dépasser ces conceptions dualistes.

(35) J'ai utilisé précédemment une traduction de Coste revue au début du XIX è siècle. J'utilise ici une traduction même de Coste, en l'occurrence celle de MDCCLV, 1755, à Amsterdam chez Mortier, qui est selon Balibar (1998: 14, note 11) sa «cinquième édition »; reprise (Naert éd.) en 1972 à Paris chez Vrin, c'est celle qu'utilisent aussi Ferret (1993, 1996), Ricœur (1990), et donc à Balibar (1998, qui cite Coste page 113); mais il en propose sa propre traduction (avec le texte original en regard) : "[W]e must consider what person stands for ; which, I think, is a thinking intelligent being, that has reason and reflection, and can consider it self as it self, the same thinking thing in different times ans places"; « [I]1 nous faut considérer ce que représente la personne ; c'est, je pense, un être pensant et intelligent, doué de raison et de réflexion, et qui peut se considérer soi-même comme soi-même, une même chose pensante en différents temps et lieux » (éd. citée : 148-149).

(36) Ce terme qui, comme on 1'a dit, est la traduction littérale de sameness, viendrait de Voltaire qui précisément, dans son Dictionnaire philosophique (1764), expose les thèses de Locke. 
Pour Wiggins (1980), il y a deux sortes d'essence : d'un côté la triviale, de l'autre la sortale; la première est définie comme l'identité qu'un objet entretient avec soi (propriété particulière) ; l'autre (générique) sert à classer l'individu sous un terme «ultime», à fixer sa référence par rapport à une espèce ; et Wiggins (op . cit. : 121) fait remarquer que la «nécessité essentielle» surgit précisément à cette limite ( « ce point d'altérabilité ») où le retrait des traits en question détruit le «porteur» lui-même » (trad. Engel 1989 : 248).

Kripke partage cet « essentialisme individualiste »-selon 1'expression d'Engel (1985 : 112 ; ses italiques) - en ce qu'il refuse de souscrire à une métaphysique des « particuliers nus », c'est-à-dire des objets sans propriétés.

Kripke est un logicien modal, c'est à dire un logicien des mondes possibles; et dans ces mondes ([1980] 1982:41):

« certaines propriétés d'un objet peuvent lui être essentielles, en ce qu'il n'aurait pu manquer de les avoir. »

Quant aux propriétés qui ne sont pas essentielles, elles sont dites accidentelles:

« Pour identifier les individus d'un monde possible à un autre, il semble que nous devions postuler certaines propriétés telles que s'ils les ont dans un monde possible, ils les ont dans tous les autres, et des propriétés que les individus ont dans certains mondes possibles, mais pas dans d'autres, c'est-à-dire des propriétés essentielles et des propriétés accidentelles ${ }^{(38)}$. $($ (Engel, $1985: 108)$

Cette essence des objets, qui supplée nos facultés de (re)connaître, c'est à dire nous impose des contraintes dans la conception des mondes possibles, repose sur trois sortes de propriétés non triviales, en dehors de la propriété générale (ou précisément triviale) de l'identité de soi à soi (国 L'Identité «triviale » II) :

- l'origine: si telle personne est telle (par exemple la reine d'Angleterre), il n'est pas possible qu'elle ait eu une autre origine, d'autres parents que ses parents (ces parents-là) ;

- la substance : si un objet est de telle matière, il ne serait plus tel (cet objet) s'il était de telle autre matière ;

- enfin, la forme (ou identité générique de l'objet) : elle équivaut à l'identité sortale présentée ci-dessus.

La première de ces propriétés (l'origine en l'occurrence des personnes), conduit à ce que Ferret (1993: $24 s q$.) appelle des «vagabondages biographiques », c'est-àdire à envisager l'identité personnelle (comme notion ou en théorie) ou encore à la projeter (individuellement ou particulièrement) dans et à travers des mondes possibles; ces «vagabondages» font partie de ce que Ferret appelle des puzzling cases, ces derniers lui permettant de faire de la "métaphysique-fiction " ( $\leftrightarrow$ Puzzling cases \& vagabondages biographiques).

(37) Je reprends et j'adapte ici Achard-Bayle (2001: 51-52).

(38) Note d'Engel : «R. Chisholm [...] 1967.» 


\section{Puzzling cases (I) : vagabondages biographiques et métaphysique-fiction ${ }^{(39)}$}

La question des «vagabondages biographiques » est en fait double et consiste à (se) demander (avec Ferret, $1993: 24$ ) :

« [J]usqu'à quel pointje pourrais être quelqu'un d'autre (biographie alternative) et jusqu'à quel point quelqu'un d'autre pourrait être moi (biographie parallèle). »

Selon Ferret, qui partage ainsi 1'essentialisme de Kripke (吗 Essences triviales et non triviales), une biographie ne concerne donc ne définit pas, métaphysiquement, une personne.

On ne saurait pour autant soutenir la thèse radicale que rien n'est essentiel à / chez une personne ; il y a précisément des « bornes strictes » à ces vagabondages biographiques, qui sont des «nécessités essentielles » auxquelles on ne peut « échapper» : l'origine et l'espèce sont ainsi deux essences ; l'une est individuelle, l'autre partagée :

« [Elles] constituent le cadre métaphysique à l'intérieur duquel les personnes, comme d'ailleurs les autres catégories de particuliers, peuvent se déployer sans que jamais soit rompue ou altérée leur identité. » $(\text { Ferret, } 1993: 27)^{(40)}$

Dans cet essentialisme, 1'appartenance à une sorte ou espèce est la première des nécessités qui conditionne et contraint la caractérisation de l'identité, sous ses deux principales formes : l'identité spécifique ou sortale déterminant l'identité numérique (par exemple, pour être cet homme, il faut au moins être un homme).

Enfin, cette propriété essentielle (par exemple « être $u n$ homme») peut se comprendre de deux manières : (i) hors du temps, dans la mesure où, par exemple, Kant n'aurait pas pu ne pas être un homme, et (ii) dans le temps, dans la mesure où, par exemple, Kant, au cours de sa carrière, n'aurait pas pu devenir un non-homme; en effet si Kant, toujours selon Ferret (1993 : 34), s'était transformé en alligator après avoir absorbé une potion magique, il aurait perdu du même coup son identité.

\section{Puzzling cases (II) : expériences et transgressions des conventions}

Il faut commencer par préciser que les expériences de pensée que sont les puzzling cases ont une longue tradition qui permet de remonter à Platon. Rosset ([1984] 1993 : 85) reprend ainsi longuement le dialogue entre Socrate et Cratyle pour sa démonstration de l'artificialité de l'identité :

«Y aurait-il deux objets, tels que Cratyle et l'image de Cratyle, si quelque divinité, non contente d'imiter ta couleur et ta forme, comme les peintres, reproduisait aussi tout l'intérieur de ta personne, tel qu'il est, lui donnait la même mollesse, la même chaleur, et y mettait le mouvement, l'âme, la pensée, tels qu'ils sont en toi, en un mot plaçait à côté de toi un double de toutes tes qualités ? Y aurait-il, en ce cas, Cratyle et une image de Cratyle, ou deux Cratyles ? » ${ }^{(41)}$

(39) Je reprends et j'adapte ici Achard-Bayle (2001:52-53).

(40) Ces deux «nécessités essentielles » rejoignent les «propriétés» de Wiggins (㖊 Essences triviales et non triviales) : la «particulière » et la « générique » (essence «individuelle», essence «partagée » chez Kripke).

(41) Platon, Cratyle (431e-432d, éd. citée : 460). 
Ces puzzling cases ont notamment pour fonction, et notamment en philosophie analytique, de «rompre avec la logique de l'identité » et à « réviser nos croyances en ce domaine » (Ferret, 1993 : 23), en proposant des fictions ${ }^{(42)}$ de duplication, transplantations, décérébration et autres téléportations ou déménagements d'âme.

Il existe ainsi des cas, plus ou moins imaginaires ou imaginables, de changement numérique, de « saut sortal » (Ferret, 1996 : 40), où sont mis à mal les principes ou les conventions ontologiques ; et, sachant que c'est aussi par l'identification, par une ou des désignations sortales que se détermine l'appartenance à l'espèce, où sont également mis à mal les principes ou les conventions linguistiques de cette identification.

Les dédoublements et les métamorphoses littéraires ( Cas et traitements littéraires de métaphysique fiction) sont autant de ces sauts, numériques et sortaux, qui incitent à voir ou revoir ces principes et conventions. Mais les philosophes contemporains, aux marges de la science-fiction, ont aussi inventé des puzzling cases susceptibles d'illustrer et d'alimenter la réflexion sur l'identité et ses évolutions. La question qu'ils se posent et veulent exposer n'est plus alors celle de l'appartenance à une espèce mais celle du changement (sortal) d'espèce ou (numérique) dans l'espèce ; ce qui est une manière de compléter le concept d'identité diachronique (吼 Identité diachronique) par la notion évolutive ${ }^{(43)}$ de carrière :

«Supposons que quelque particulier doive devenir tout d'un coup roi de la Chine, mais à condition d'oublier ce qu'il a été, comme s'il venait de naître tout de nouveau ; n'est-ce pas autant dans la pratique, ou quant aux effets dont on se peut apercevoir, que s'il devait être anéanti et qu'un roi de Chine devait être créé dans le même instant à sa place ?» (Leibniz,Discours de métaphysique, éd. citée : article 34$)^{(44)}$

Si l'on distingue généralement les particuliers naturels (et pas seulement personnels) des artefacts, il n'en reste pas moins que l'unité de la carrière des uns et des autres est intrinsèquement liée à leur permanence structurelle, qui font d'eux, organismes ou objets, des totalités ; toutefois le changement (naturel ou qualitatif) est indispensable au maintien de l'identité des premiers, alors qu'il n'est pas nécessaire aux seconds, même si l'on considère que l'usure et la réparation font «naturellement » partie de leur carrière.

\section{Le bateau de Thésée}

On peut alors évoquer le paradoxe du bateau de Thésée, tout d'abord suivant Plutarque :

«Le vaisseau sur lequel Thésée s'était embarqué avec les autres jeunes gens, et qu'il ramena heureusement à Athènes, était une galère à trente rames, que les Athéniens conservèrent jusqu'au temps de Démétrios de Phalère. Ils en ôtaient les vieilles pièces, à mesure qu'elles se gâtaient, et les remplaçaient par des neuves qu'ils joignaient solidement aux anciennes. Aussi les philosophes, en disputant sur ce genre de sophisme qu'ils appellent croissant, citent ce vaisseau

(42) Quelle que soit la part du littéraire dans ces expériences, Ferret (op. cit. : 99) demande de ne pas confondre fantastique et logiquement impossible.

(43) Par cet adjectif, je fais allusion à tous les travaux sur les référents évolutifs des années 90 (pour un panorama, $c f$. Achard-Bayle, 2001, particulièrement dans la " petite grammaire des métamorphoses » finale : 253-268).

(44) Voir aussi Mallarmé Contes indiens, in Euvres complètes, éd. citée. 
comme un exemple de doute, et soutiennent les uns que c'était toujours le même, les autres que c'était un vaisseau différent. "(Plutarque, Vie des hommes illustres, éd. citée, en ligne)

Le cas est repris par Hobbes qui se demande :

«[...] si deux corps existant tous deux en même temps seraient un même corps. Car si par exemple, ce bateau de Thésée, concernant la différence introduite par une réparation continue en retirant les vieilles planches et en en mettant de nouvelles, dont les sophistes d'Athènes étaient si enclins à discuter, était, après le changement de toutes les planches, le même bateau numériquement que celui qui était au début; et si un homme avait gardé les vieilles planches au moment où elles étaient retirées, et en les rassemblant ensuite dans le même ordre, avait encore une fois construit un bateau à partir de celles-ci, celui-ci sans doute devrait aussi être numériquement le même bateau que celui qu'il y avait au début, et ainsi il y aurait deux bateaux numériquement identiques, ce qui est absurde. » (Hobbes De Corpore, II, II in Engel \& Nef, 1988 ; on trouvera une autre traduction in Ferret, 1996:108)

Le problème posé ainsi par les particuliers (naturels ou artificiels) est qu'ils peuvent changer, mais seulement jusqu'à un certain point. Et c'est dans la détermination de ce seuil, de cette limite, que réside la difficulté : là, « il y a un peu de vague », note Kripke ([1980] $1982: 39)$.

Mais on ne saurait s'en tenir à ce constat, car la philosophie contemporaine apporte du nou veau à la tradition logique ou métaphysique au moins sur deux points : d'une part, elle opère la distinction entre particuliers naturels et artefacts; $d$ 'autre part, elle dirige l'attention autant, sinon davantage, sur la détermination de l'identité et du seuil de changement que sur l'identité en soi.

Pour les artefacts, l'invariance et l'unicité compositionnelle ne sont, selon Ferret (1996: 100-102), ni nécessaires (i) ni suffisantes (ii) :

(i) « un seul et même particulier peut très bien ne pas être constitué des mêmes composants à deux moments de sa carrière »,

(ii) «deux particuliers peuvent être composés des mêmes composants », comme un lit transformé, i.e. réassemblé, en bibliothèque).

Ainsi pour Ferret (ibid.) :

(i) « un artefact peut subir certains changements de composition tout en demeurant un et le même » et

(ii) « un artefact qui subit un changement complet de tous ses composants n'est plus un et le même.»

Reste que le seuil du changement n'est pas pour autant déterminé : est-il même déterminable? Selon Ferret (ibid.) :

« [I]l n'est certainement pas très sérieux de prétendre trouver la limite ultime. »

Ceci ne doit pas nous ramener pour autant à la thèse de l'identité comme fiction, car, selon Ferret (op. cit. : 117),

« s'il est vain de vouloir déterminer un seuil de substitution, la limite n'est pas une chimère. »

La difficulté est ailleurs, moins dans l'indétermination de l'identité, ou d'une 
identité, que dans notre indétermination devant une certaine identité, ou une identité à un certain moment : ainsi, précise Ferret (op. cit. : 135) :

« [L]e fait que nous puissions être dans l'expectative pour énoncer un jugement d'identité n'entraîne aucunement que l'identité elle puisse être indéterminée. »

Ceci appelle la remarque d'Engel \& Nef (1988: 492), pour qui il peut y avoir « des énoncés d'identité vagues (relativement à notre connaissance), sans que les objets eux-mêmes soient vagues ».

Le vague est alors, non pas dans les choses, de re, mais relatif à notre connaissance et à notre langage, de dicto.

\section{Cas (et traitements) littéraires de métaphysique fiction}

Pour les particuliers personnels, voire plus généralement tous les particuliers ou individus conscients, le seuil ou la limite du changement est la conscience, ou, en termes moins spiritualistes ou mentalistes, le cerveau qui en est le siège. Tel est le " critère cérébral » défini par Ferret dans son premier ouvrage (1993, dont il n'est pas inutile de rappeler ici le titre : Le Philosophe et son scalpel ${ }^{(45)}$.

Pour en éprouver la validité, on a tout intérêt, là encore, de recourir aux hypothèses de la métaphysique-fiction. Or nombreuses sont les expériences littéraires de « chirurgie » mentale, d' Avatar de Gautier à La Machine de Belletto qui le confirment : une personne dont le cerveau a été « transféré » se trouve en quelque sorte « transférée » avec lui.

À l'inverse, une personne qui se métamorphose en un spécimen d'une espèce animale est effectivement métamorphosée si et à partir du moment où elle perd la conscience de soi et de son humanité :

(1) «Voilà maintenant quatre jours qu'elle jeûne... Les deux premiers jours, l'âme humaine était dans toute sa vigueur... Elle me suppliait, elle m'implorait. [...] Le troisième jour, qui était donc hier, mercredi, l'âme humaine disparut complètement... Le chat sortit ses griffes, il avait faim... Ses dents devenaient longues... Il se prit à miauler, à hurler... ${ }^{(46)}$

(2) «Mais moi, bien que je fusse devenu un âne en tout et, de Lucius, une bête de somme, je conservais pourtant une intelligence humaine. Ainsi je réfléchis longtemps et profondément...» ${ }^{(47)}$

Dans le texte (1), où l'on assiste à la métamorphose d'une marchande de gâteaux en chat, le changement se traduit également au niveau linguistique ; et ce n'est même qu'à ce niveau-là qu'il est interprétable comme tel par le lecteur; autrement dit, la fiction et son processus d'évolution contrefactuel ne sont « possibles » c'est-à-dire effectifs dans la fiction qu'à partir du moment où la narration, ou plus exactement encore, où ou le discours narratif enregistre de dicto ce qui se passe de re (même si le monde de re en question est un monde de pure fiction). Du point de vue de la représentation et de l'organisation textuelles, le processus méta-

(45) «Le critère ultime de l'identité personnelle n'est rien d'autre que la cause originale des événements mentaux », autrement dit le cerveau (Ferret, op. cit. : 78).

(46) Erckmann-Chatrian, Les trois âmes, in Contes fantastiques complets, éd. citée : 433-434. La ponctuation, sauf les pointillés encadrés, est celle du texte.

(47) Apulée, L’Âne d'or ou les métamorphoses, éd. citée : 88. 
morphique entraîne ainsi un changement des chaînes de référence, et de ce fait de désignation sortale : la marchande lelle... $\rightarrow$ le chat / il...

Ceci dit, pour revenir à nos considérations métaphysiques inspirées de la « chirurgie cérébrale » de Ferret (1993), il est à noter que les changements en question, de re et de dicto, ne s'effectuent qu'après que l'âme a disparu, et seulement alors. De ce point de vue, le texte (2) est à considérer comme un contre-exemple : il est en effet un récit de métamorphose " de pure forme », en ce qu'il ne concerne que l'apparence ou si l'on veut le contenant, puisque l'activité cérébrale reste la même au travers de la même conscience qui continue de réfléchir; du même coup continue d'apparaître le marqueur personnel d'autodésignation je qui est bien une marque irréductible de l'identité ${ }^{48)}$.

Sorties de la dimension mentale ou cérébrale, autrement dit confrontées avec le physique ou la matérialité des particuliers, leur forme apparente ou même leur structure cachée, les choses, paradoxalement, se compliquent ; " paradoxalement ", dis-je, car on pouvait imaginer, en tout bon sens, ou suivant le sens commun, que le mental est d'une bien plus grande complexité ! La fiction tend à montrer le contraire...

Pour ce qui est de l'apparence ou du phénotype, sa conservation bloque le processus de transformation en une autre espèce ou substance, du moins des points de vue cognitif ou linguistique, comme ici dans les paroles ou les pensées d'un personnage témoin de la métamorphose :

(3a) «Je passai ensuite dans une chambre très richement meublée, où j'aperçus une dame aussi changée en pierre. Je connus que c'était la reine...»

(3b) «Le roi mon père éprouva le même sort : il fut métamorphosé en une pierre noire, tel qu' on le voit dans un endroit de ce palais... ${ }^{(49)}$.

La question est plus complexe encore avec la structure cachée, l'organisme ou l'organisation biologique des particuliers. Ferret (1996:39-40, note 11) reprend et commente ainsi le puzzling case imaginé par Price (1977) :

(4) Un chien, Rover, fait un voyage dans l'espace ; revenu sur terre, il devient progressivement une masse informe de cellules, Clover, qui, bien qu'elle lui soit continue spatio-temporellement, n'a plus, du chien, ni la forme ni le code génétique.

Pour la philosophe américaine il y a continuité, donc identité entre Rover et Clover. Ferret quant à lui refuse cette identité dès lors que le saut sortal ${ }^{(50)} \mathrm{a}$ eu lieu, car pour être ce chien il faut encore être $u n$ chien. Il en conclut que « le problème n'est donc pas de savoir si Rover et Clover désignent le même chien » pour orienter sa réflexion vers ce vague dont nous parlions (咜 Le Bateau de Thésée) qui serait ici « une période indéterminée au cours de laquelle nous ne savons plus à quel être nous avons affaire» (ibid.).

(48) Cf. Ricœur (1990: 61 sq.) et mes propres développements : Achard-Bayle (2001 : 165-171, $\S$ «La résistance au changement : le rôle des pronoms, il vs je »).

(49) «Histoire de Zobéide», éd. citée : 208 et 212.

(50) Ou « changement de type 2 » («de nature» vs « changement de type 1 [...] de degré») qui « rompt» (vs qui «préserve ») l'identité (Ferret, 1996: 32 ; italiques de l'auteur). 
Certes, les cas ne sont pas rares où, pour les êtres monstrueux, au sens sortal de "dégénérés ", on doive recourir à des dénominations complexes ${ }^{(51)}$, de la légendaire Mélusine femme-serpent aux hybrides hommes-singes créés « scientifiquement» par le Docteur Moreau.

Mais je ferai remarquer que, si le vague est bien une question de dicto, il n'y a alors de «période indéterminée », ou de période d'indétermination, que tant qu'un nouvel acte de baptême n'a pas eu lieu. Ainsi, pour reprendre le cas posé par Avatar, il n'y a de période d'hésitation ou d'indétermination chez le narrateur ou le lecteur de ce récit qu' aussi longtemps que les deux personnages, dont le docteur Cherbonneau déménage les âmes, ne sont pas rebaptisés :

(5a) " "Réveillons nos dormeurs," dit M. Cherbonneau [...] et se plaçant devant le corps du comte Labinski habité par l'âme d'Octave, il fit les passes nécessaires pour le tirer de l'état somnambulique. [...] Au bout de quelques minutes, OctaveLabinski (désormais nous le désignerons de la sorte pour la clarté du récit) se redressa sur son séant [...] » (éd. citée : 260 ; je souligne).

(5b) «Resté seul avec le corps d'Octave de Saville, habité par l'âme du comte Olaf Labinski, le docteur Balthazar Cherbonneau se mit en devoir de rendre cette forme inerte à la vie ordinaire. Au bout de quelques passes, Olaf-de Saville (qu'on nous permette de réunir ces deux noms pour désigner un personnage double) sortit comme un fantôme du profond sommeil...» (éd. citée : 265 ; je souligne).

Or, en l'occurrence ce « longtemps » est, narrativement, court...

Pour revenir au cas précédent, et pour aller au-delà du problème de l'identité sortale ou non des deux états ou entités désignés, Rover/Clover, je remarque que ni Price ni Ferret ne s'arrête sur le fait qu'il a été nécessaire de rebaptiser (en Clover) le premier particulier (Rover). Or ce changement d'identité (ici au sens administratif du terme $)^{(52)}$ est bien le signe - ou le symptôme dans les termes de Ferret - qu'il n'est plus possible de désigner de la même manière ce référent évolutif. Ce qui veut bien dire que l'identité est aussi une question nominale, que l'identité est aussi nominale.

On voit également que, s'il peut être «vain » de chercher à savoir quand s'effectue le saut sortal, il peut être utile de porter l'attention sur les indices discursifs de cette opération. C'est ce que les linguistes de la référence évolutive (Charolles, Kleiber, Schnedecker... $\left.{ }^{(53)}\right)$ ont proposé de faire dans les années $90 \ldots$

Je ne pense pas me tromper si, pour finir, j'ajoute que ce sont les fictions qui pour une grande part ont déclenché la recherche sur les référents évolutifs, et permis de poser et d'affiner sa ou ses problématiques. C'est en tout cas le sens que j'ai voulu donner à ma Grammaire des métamorphoses : ce sont en grande part les cas de fiction, tels qu'ils sont mis en discours, donc ces cas avec leurs marques textuelles qui m’ont servi à élaborer cette grammaire du changement.

(51) Ferret, qui se réfère là à Wiggins (1980:67), parle lui de «prédicats mixtes ».

(52) $C f$. la conception " antinaturaliste » que défend Rosset ([1984] 1993) : l'identité est une identité de papiers.

(53) Je ne peux les citer tous, ni citer tous les travaux de chacun; je renvoie pour cela à ma bibliographie de 2001, telle notamment que je la commente dans mon post-scriptum (pp. 271273). 


\section{Conclusion. L'Identité et le bon sens}

Comme annoncé, pour compléter le panorama des fondements de l'identité logique, voici les « lois de Leibniz " (吼 Relativité de l'identité I). Mais c'est aussi par là que tout aurait pu ou pourrait commencer, car il s'agit bien de fondements, logiques et chronologiques...

«Il n'y a, semble-t-il, qu'en logique qu'on trouve des énoncés de la forme $a=a$ ou $x=$ y. » (Engel, $1989: 238)$.

C'est pourtant bien ce qui fonde la logique classique, et que l'on trouve, précisément, dans les «lois de Leibniz»:

- L'indiscernabilité des identiques, tel que si A et B sont identiques, alors tout ce qui est vrai de A est vrai de B («ils sont indiscernables » veut dire qu'ils ont toutes leurs propriétés en commun);

- L'identité des indiscernables, tel que si tout ce qui est vrai de A est vrai de B, alors A est identique à $\mathrm{B}$ (ce qui revient à dire que si deux objets ont toutes leurs propriétés en commun, alors ils sont identiques) ;

- La substituabilité des identiques ou de substitution salva veritate - que Leibniz définit ainsi : «Les termes dont l'un peut être substitué à l'autre sans affecter la vérité des propositions dans lesquelles ils figurent sont identiques ${ }^{(54)}$. »

En fait l'étrangeté des « énoncés d'identité » (Engel, ibid.) $a=a$ ou $x=\mathrm{y}$ vient de ce que l'identité y est et n'y est envisagée que de manière absolue.

"Les énoncés d'identité ordinaires sont plutôt du genre : "Cette table est-elle identique à celle-ci ?" ou "Cethomme est-il le même que celui-là ?" ( (Engel, ibid.)

Et encore faudrait-il contextualiser : "Cette table est identique en tous points, forme, matière... à celle-ci », "Cet homme, sur cette photo ${ }^{(55)}$, est le même que celui sur cette autre photo, prise vingt ans auparavant. »

Autrement dit, on y revient, ces énoncés d'identité laissent entendre en fait qu'un objet ou qu'un individu ( Individus \& particuliers I \& II) est identique à, ou est le même qu'un autre en fonction d'une ou de plusieurs propriétés : propriétés matérielles formelles, ou constitutives (婙 Essences minimales, 国 Qualités premières et secondes), apparence ou ressemblance (国 Apparences I \& II), continuité dans le temps ( Identité diachronique, Identité dans le temps)...

Ce qui nous ramène à l'identité relative (吗 Identité relative I \& II).

Or, ce sont aussi précisément ces équations ou relations et principes d'identité décntextualisés ou pour tout dire, "vulgairement», i.e. dans le langage du commun, « déconnectés » du réel et précisément du langage quotidien que dénonce le célèbre aphorisme de Wittgenstein ([1922] 1993 : 88) :

«Sommairement parlant, dire que deux choses sont identiques est dépourvu de sens, et dire d'une chose qu'elle est identique à elle-même, c'est ne rien dire du tout. $\gg(56)$

(54) Cité par Engel (1989: 232).

(55) On touche là à une autre problématique sémantique, inspirée de la pragmatique : les espaces mentaux (Fauconnier, 1984).

(56) Tractatus logicus philosophicus, § 5.5303 (trad. Granger). 
Plus radicalement encore, on pourrait finir avec cet autre aphorisme :

«Sur ce dont on ne peut parler, il faut garder le silence. » (Wittgenstein, § 7, [1922] éd. citée $1993: 112$ )

\section{Pour en savoir plus. Varia}

Je termine par une série d'entrées (beaucoup) plus brèves, à lire comme autant de nota bene, voire de compléments bibliographiques... Je les « repousse » ainsi in extremis, car les questions que j'y traite ne relèvent pas (strictement) de la problématique de l'identité. Pour autant, les textes auxquels je renvoie dans la plupart des cas illustrent parfaitement à un titre ou un autre (voire a contrario pour Métaphore et sens figuré) la thématique générale de ce numéro de Pratiques.

\section{Métaphore et sens figuré (Le poids de la tradition scolaire et de la métalinguistique ordinaire sur la formation des concepts de métaphore)}

Je consacre quelques pages de mon ouvrage (2008 : 123-124) à présenter et discuter une théorie radicalement constructiviste et antiréférentialiste de la métaphore et du sens figuré : il s'agit notamment pour Schulz (2002), qui s'oppose à la notion même de sens figuré, de savoir si l'usage métalinguistique si fréquent du mot «métaphore » n'est pas un effet de la tradition « référentialiste »-autrement dit : largement répandue, commune, non savante, ordinaire ? -, sur la formation (ou la « déformation ») des sujets parlants eux-mêmes.

«Rares sont en linguistique les termes qui, comme la métaphore, bénéficient d'une telle notoriété au-delà même des sciences du langage. Les sujets parlants "naïfs" mêmes semblent en effet en faire un usage fréquent. [...] Mais s'agit-il d'un usage analogue à celui fait par les linguistes ? »(Art. cité, début de l'introduction : 21)

« Notre point de départ était [...] le constat d'un usage métalinguistique du mot "métaphore" chez les sujets parlants. Contrairement à ce qu'un tel usage et, plus généralement, une croyance solidement enracinée pourraient faire croire, la métaphore est non pas un "phénomène objectif", existant réellement dans la langue, mais elle n'est qu'un "phénomène", ou, plus exactement, un fait, c'est-à-dire une entité jugée linguistiquement pertinente. Elle implique, de ce fait, un certain nombre d'hypothèses que nous avons essayé de rendre explicites : en se construisant sur un dédoublement des emplois, la métaphore se donne à voir sur un fond de description référentialiste, réduisant la langue à une image du monde. La métaphore n'existe que si on suppose comme inhérentes à la signification des mots des propriétés relatives aux objets du monde, auxquelles ces mots auraient pour fonction de renvoyer.

En fait, sujets parlants et linguistes - considérés dans la perspective de la métaphore - partagent la même croyance, la même image de la langue. Introduire un sens métaphorique a la même fonction pour les deux : c'est élever l'impression d'une différence au niveau d'un fait; les sujets parlants adoptent le même point de vue référentialiste que les linguistes. Et on peut se demander si les premiers ne l'ont pas "hérité" des seconds : l'usage métalinguistique ne serait alors qu'un effet d'une réflexion menée sur la nature de la langue. » (Art. cité, intégralité de la conclusion : 36-37)

(Pour un point de vue différent : «Le bon sens commun ») 
Je consacre une autre partie de mon ouvrage (2008 : particulièrement 41-47) à celui de Larsson (1997) ${ }^{(57)}$ qui porte ce titre même :

"Toute théorie qui ne tient pas compte de ce fait linguistique, à savoir du sens "naturel" du mot de vérité, s'éloigne de ce fait du sens commun, et aura tendance à expliquer quelque chose qui n'a pas, justement, d'existence réelle dans la langue. Ce sera, si l'on veut, un non-sens.

[...] De la même manière, dire [...] que le monde réel n'est qu'un monde "possible" parmi d'autres est infirmé par notre manière de concevoir le monde et la réalité - et par sa traduction en termes linguistiques. En effet, l'existence communément admise d'un concept du monde réel est la preuve suffisante que ce monde-là n'est pas assimilable et équivalent à d'autres mondes seulement possibles ou imaginables. » (Larsson, 1997 : 55)

«Le bon sens commun» (les italiques sont de l'auteur) est un plaidoyer pour un équilibre - particulièrement savant - entre (i) un engagement en faveur d'une sémantique (re) connectée au réel, au nom du «bon sens » ou du « sens commun » (cf. citation supra), (ii) la prise en compte des subjectivités propres aux (innombrables) interactions, (iii) sans oublier que ces subjectivités (pour fonctionner) convergent en des collectivités ou précisément des communautés (intersubjectives) de (construction, d'établissement du) sens commun.

Et puisque nous voici sur l'intersubjectivité, on ne s'étonnera pas de voir Kleiber entrer en lice, et citer lui-même (2001) Larsson :

« [C]e qui prévaut pour et dans le langage [c'est] le sens commun de vérité. Larsson [op. cit. : 55] a, en effet, tout à fait raison de souligner que l'on ne peut expulser la notion de vérité de la sémantique linguistique tout simplement parce que nous l'utilisons pour précisément indiquer si ce qui est exprimé par telle ou telle phrase est vrai ou faux par rapport à (ce que nous croyons être ou ce que nous savons de) la réalité : "Si par exemple nous analysons le sens du terme de vérité, il faut tenir compte du fait que la notion de vérité existe bel et bien dans la langue naturelle et qu'elle est effectivement employée par n'importe qui pour mesurer l'adéquation des propositions par rapport aux choses (concepts, etc.) dans ce que nous appelons le monde réel extralinguistique" [Larsson, ibid.]. » (Kleiber, op. cit. : 357)

Pour le reste, je renvoie à Kleiber et à ses autres ouvrages déjà cités (1997, 1999).

\section{«La Pensée sauvage »}

Ouvrage de Lévi-Strauss ([1962] 1990), où il paraît d'emblée (première page du premier chapitre : «La Science du concret ») que ladite pensée est tout sauf «primitive » :

« [...] prétendue inaptitude des "primitifs" à la pensée abstraite [...]. C'est ainsi que le chinook, langue du nord-ouest de l'Amérique du Nord, fait usage de mots abstraits pour désigner beaucoup de propriétés, ou de qualités des êtres et des choses: "Ce procédé, dit Boas, y est plus fréquent que dans tout autre langage connu de moi." La proposition : le méchant homme a tué le pauvre enfant, se rend en chinook par : la méchanceté de l'homme a tué la pauvreté de l'enfant [...] (Boas [1911 :] 657).» (Lévi-Strauss, op. cit. : 11)

(57) Je n'ai pas pu prendre en compte Larsson (2008) sorti trop tard. 


\section{Épidémie / Contagion des idées}

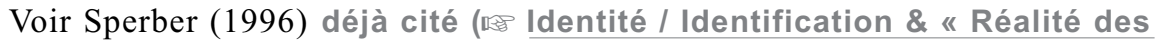
espèces $॥$ ).

\section{Tertium datur}

Absurdité logique, le tertium étant par définition non datur! Qu'est-ce à dire ? Qu'une entité ne peut pas être et ne pas être en même temps, autrement dit l'un et l'autre, vrai ou faux... Mais du tiers exclu des Grecs et de l'être ou ne pas être d'Hamlet au soi-même comme un autre de Ricœur (1990), que de chemin parcouru!

La fiction propose des cas de tertium datur : «C'est ma chambre et ce n'est pas ma chambre » (䟚 L'Identité triviale « revue à la baisse »); c'est pourquoi j' ai pour ma part sous-titré mon étude d'Avatar ( Cas (et traitements) littéraires de métaphysique fiction) : "Vers une logique du tertium datur? » (Achard-Bayle, 1997).

\section{Le Linguiste et le peuple}

J'ai brièvement évoqué la question dans mon hommage à Mistral et à sa prose d'almanach ${ }^{(58)}$. Sinon, la question a longuement été débattue par le collectif Pratiques lorsque les éditeurs du présent recueil sont venus lui présenter ce sommaire. Pour nous, populaire n'est pas lié à peuple (en tant que son dérivé). Nous ne nous intéressons donc pas au «peuple» pour lui-même; si nous nous intéressons à lui, ce n'est qu'indirectement : la linguistique dont on débat ici-même, et après quelques autres, est populaire certes, mais (d'où notre titre interrogatif) sans doute aussi naïve, profane, laïque, ludique, ordinaire, commune, et même parfois plus savante ou érudite que scientifique.

\section{The Literary Mind}

Les linguistes cognitivistes américains - autrement dit les CogLingers californiens : Lakoff et Turner notamment -, ont plaidé, sinon pour une linguistique expressément populaire, du moins pour une linguistique de l'esprit littéraire ordinaire. Pour Lakoff \& Johnson ([1980] 1985), ce sont les métaphores qui témoignent de cet esprit, « dans la vie quotidienne »; pour Turner, ce sont nos facultés de (nous) représenter les événements comme autant de récits, tout comme notre habitude de (nous) représenter les entités par diverses sortes de mélanges, au titre desquels de nouveau les métaphores (voir ses nombreux travaux avec Fauconnier sur le blending, cf. Fauconnier \& Turner ) mais aussi la création d'hybrides fabuleux (Literary Mind, 1996).

\section{Logique \& Divertissement. Bon sens \& Justesse de l'esprit. Pour une éducation de l'esprit juste, linguistique... et ordinaire ?}

Au siècle de Pascal, Arnauld \& Nicole ([1662] 1970) écrivent :

«La naissance de ce petit ouvrage est due entièrement au hasard \& plutôt à une espèce de divertissement [...]. Il n'y a rien de plus estimable que le bons sens \& la

(58) Introduction: «[Pichoto] publicacioun, souto formo d'armana » et note 1. 
justesse de l'esprit dans le discernement du vrai \& du faux. [...] On se sert de la raison comme d'un instrument pour acquérir les sciences $\&$ on se devroit servir au contraire des sciences comme d'un instrument pour perfectionner la raison. [...] Si l'on ne s'y applique dans ce dessein, on ne voit pas que l'étude de ces sciences spéculatives, comme de la Géométrie, de l'Astronomie \& de la Physique, soit autre chose qu'un amusement assez vain. [...] Non seulement ces sciences ont des recoins \& des enfoncements fort peu utiles; mais elles sont toutes inutiles, si on les considère en elles-mêmes \& pour elles-mêmes [ Wittgenstein L'Identité et le bon sens]. Les hommes ne sont pas nés pour employer leur temps à mesurer des lignes [...] mais ils sont obligés d'être justes, équitables, judicieux dans tous leurs discours [...] c'est à quoi ils doivent particulièrement s'exercer \& se former. » (op. cit. : 29 et 35-36).

\section{Bibliographie}

ACHARD-BAYLE, G. (1996) : «La désignation des personnages de fiction : les problèmes du nom dans François le Champi », Poétique 107, 333-353.

— (1997) : "Sémantique et pragmatique de la référence évolutive ; parcours théoriques et étude de cas : vers une logique du tertium datur? », Revue de Sémantique et Pragmatique 2, Orléans, Sup' Or, Presses Universitaires, 63-89.

- (2001) : Grammaire des métamorphoses, Bruxelles, Duculot De Boeck Université.

— (2006) : « De la mythologie à la chirurgie plastique : résistance, vacance et perte du nom propre ", Actes de la journée Nom propre en discours, Paris 3, 12 oct. 2006, à par. in Carnets du Cediscor, Paris, Presses Sorbonne nouvelle.

— (2007) : "Principes - savants - d'identité “ triviale” », communication au colloque international Construction d'identité et processus d'identification, Tours, 29-30 novembre 2007, à par. in Actes.

- (2008) : Les Réalités conceptuelles. Identitéet / en Fiction, Metz, CELTED, Coll. Recherches textuelles 8 .

ARISTOTE [1991 pour l'éd. et la trad. fr. utilisée] : La Métaphysique, trad. J. Barthélemy Saint-Hilaire, P. Mathias \& J.-L. Poirier (éds), Paris, Press Pocket.

- De la génération et de la corruption (cité par Ferret, 1996 : 38).

- Poétique, [1990 pour l'éd. et la trad. fr. utilisée], M. Magnien (éd.), Paris, Librairie générale Française, LGF, Le Livre de Poche Classique.

ARNAULD, A. (1660) : Grammaire générale \& raisonnée, éd. numérisée du site Gallica, Paris, BNF.

A RNAULD, A. \& NiCOLE, P. ([1662] rééd. 1970) : La Logique ou l'art de penser, Paris, Flammarion, coll. Champs.

Bettelheim, B. ([1976] trad. fr. et rééd. 1990) : Psychanalyse des contes de fées, Paris, Hachette-Pluriel.

BoAs, F. (1911) : « Handbook of American Indian Language Part I », Bulletin 40, Bureau of American Ethnology, Washington, Government Print Office, Smithsonian Institution, Bureau of American Ethnology (cité par C. LéviStrauss [1962] rééd. 1990:11).

BOÈCE, cité par Ferret (1993: 21, sans autre mention).

Bromberger, C. (1982) : «Pour une analyse anthropologique des noms de personnes », Langages 66, 103- 124. 
Charolles, M. (1997) : «Lecture et identification des personnages dans les récits de métamorphose », Problèmes de lecture 1, L'interprétation, Université de Reims, 125-161.

CHISHOLM, R. (1967) : «Identity through possible worlds, some questions », NOUS $1,1-8$.

Cure, A. (2006a) : Le Fonds Godefroy, mémoire de master 2, ss la dir. de F. Duval, Université de Metz (2 vol., 86 pages, avec annexes et illustrations hors texte). - (2006b) : La Représentation et l'étude du lexique médiéval de la fin du $X V I I^{e ̀ m e}$ siècle à 1902, projet de thèse présenté au concours des allocations de recherche de l'École doctorale PIEMES (Perspectives interculturelles : écrits, médias, espaces, sociétés), Université de Metz (5 pages).

DeSCARTES [1963-1973 pour l'éd. citée par Ferret, 1996 : 28] : Méditations II in Euvres philosophiques, F. Alquié (éd.), Paris, Garnier.

Dubois, D. (2008) : « Sens communs et sens commun : expérience sensibles, connaissance(s) ou doxa ? ", Langages 170, 41-53.

Dumont, J.-P., éd. (1991) : Les Écoles présocratiques, Paris, Gallimard, FolioEssais.

Elias, N. ([1984] trad. et éd. fr. 1996) : Du Temps, Paris, Fayard.

ENGEL, P. (1982) : «Identité, essence et modalité », Archives de philosophie, 45-3, 425-440.

— (1984) : «Identité, désignation et matérialisme », Recherches sur la philosophie et le langage 4, Grenoble, 185-217.

- (1985): Identité et référence, Paris, Presses de l'École Normale Supérieure.

- (1989) : La Norme du vrai, Paris, Gallimard.

ENGEL, P, \& NEF, F. (1988) : «Identité, vague et essences », Les Études Philosophiques 4, 475-494.

FAUCONNIER, G. (1984) : Espaces mentaux, Paris, Éd. de Minuit.

FAUCONNIER, G. \& TURNER, M. (1996) : «Blending as a Central Process of Grammar », in A. Goldberg (ed.), Conceptual Structure, Discourse and Language, Cambridge, University Press, 113-129. Version étendue en 1998, en ligne (consultée en nov. 2008) : http ://markturner.org/centralprocess.WWW/centralprocess.html.

FERRET, S. (1993) : Le Philosophe et son scalpel. Le problème de l'identité personnelle, Paris, Éd. de Minuit.

- (1996) : Le Bateau de Thésée. Le problème de l'identité à travers le temps, Paris, Éd. de Minuit.

FREGE, G. ([1892] trad. fr. et rééd. 1971) : «Sens et dénotation », in Écrits logiques et philosophiques, Paris, Seuil, coll. Points.

GEACH, P. T. (1972) : Logic Matters, Oxford, Blackwell.

HOBBES, T. [1655] : De Corpore (cité par Engel \& Nef, 1988 ; autre traduction in Ferret, $1996: 108)$

HumE, D. ([1739] trad. fr. 1968) : Traité de la nature humaine, Paris, Aubier-Montaigne.

Kleiber, G. (1997) : «Sens, référence et existence », Langages 127, 9-37. — (1999) : Problèmes de sémantique, Lille, Presses Universitaires du Septentrion.

— (2001): «Sur le sens du sens : objectivisme et constructivisme », in D. Keller et al. (éds), Percevoir : monde et langage. Invariance et variabilité du sens vécu, Sprimont (Belgique), Mardaga, 335-370. 
KRIPKE, S. ([1980] trad. fr. 1982) :La Logique du nom propre, Paris, Éd. de Minuit.

LAKOFF, J. \& JOHNSON, M. ([1980] trad. fr. 1985) : Les métaphores dans la vie quotidienne, Paris, Éd. de Minuit.

LARSSON, B. (1997) : Le bon sens commun. Remarques sur le rôle de la (re-) cognition intersubjective dans l'épistémologie et l'ontologie su sens, Lund, University Press, coll. Études romanes de Lund 47.

— (2008) : «Le sens commun ou la sémantique comme science de l'intersubjectivité humaine », Langages 170, 28-40.

LEIBNIZ, G. W. VON [2004 pour la trad. et l'éd. fr.] : Discours de métaphysique, M. Fichant (éd.), Paris, Gallimard, coll. Folio.

LÉVI-STRAUSS, C. ([1962] rééd. 1990) : La Pensée sauvage, Paris, Presses-Pocket. - (1987) : dir., L'Identité, Paris, Presses Universitaires de France, coll. Quadrige.

LOCKE, J. [1690] : An Essay on Human Understanding (diverses traductions et éditions mentionnées en notes).

LyONS, J. ([1978] 1990 pour la trad. et l'éd. fr.) : Sémantique linguistique, Paris, Hachette.

Molino, J. (1982) : «Le nom propre dans la langue », Langages 66, 5-21.

NEF, F. (1991) : Logique, langage et réalité, Paris, Les Éditions universitaires.

PlAton [1967 pour l'éd. et la trad. fr. utilisée] : Cratyle, É. Chambry (éd.), Paris, Flammarion, coll. G-F.

PRICE, M. S. (1977) : «Identity Through Time », The Journal of Philosophy 74, 201-217.

QUINE, Willard van Orman ([1960] trad. fr. 1978) : Le Mot et la chose, Paris, Flammarion.

— (1961) : From a Logical Point of View, New York, Harper \& Row.

— ([1969] trad. fr. 1977) : Relativité de l'ontologie, Paris, Aubier.

PLUTARQUE [1783-1792 pour la trad. fr. de l'Abbé Ricard] : Vie des hommes illustres, disponible sur la Toile, consulté en nov. 2008, ugo.bratelli.free.fr/Plutarque/PlutarqueThesee.htm.

PRotagoras (voir Dumont).

Putnam, H. (1975) : « The Meaning of Meaning », in Philosophical Papers vol. 2, Mind, Language and Reality, Cambridge, Cambridge University Press, 215-271.

RANK, O. ([1932] trad. fr. \& rééd. 1973) : Don Juan et Le Double. Études psychanalytiques, Paris, Payot, coll. Petite Bibliothèque Payot.

REBOUL, A. (1993) : «Le poids des pères, le choc des fils : prédicats de phase, modificateurs et identification », Cahiers de Linguistique Française, 14, 229-246.

— (1997) : «Combien y a-t-il de poulets ici ? Les référents évolutifs, identité et désignation », in G. Kleiber, C. Schnedecker \& J.-E. Tyvaert (éds.), La continuité référentielle, Metz, CELTED, Coll. Recherches linguistiques 20, 149179.

RICEUR, P. ([1983-1985] rééd. 1991) : Temps et récit, Paris, Seuil, rééd., 3 vol. coll. Points.

— (1990) : Soi-même comme un autre, Paris, Seuil.

- (1991) : «L'identité narrative », Revue des Sciences Humaines, LXXXXV, 221, 35-47.

Rosset, C. ([1984] 1993) : Le Réel et son double, Paris, Gallimard, Folio, Essais.

RouQuette, J. ([1963] $2^{\mathrm{e}}$ éd. mise à jour 1968) : La Littérature d'oc, Paris, Presses Universitaires de France, coll. Que sais-je? 
SCHULZ, P. (2002) : «Le caractère relatif de la métaphore », Langue Française 134, 21-37.

Thomas D’Aquin, Somme théologique, III, 74, III (cité par Ferret 1996 : 48, note 3$)$.

TODOROV, T. ([1970] rééd. 1990) : Introduction à la littérature fantastique, Paris, Seuil, rééd., coll. Points.

TURNER, M. (1996) : The Literary Mind, The origins of Thought and Language, New York-Oxford, Oxford University Press.

SEARLE, J. ([1996] trad. fr. 1998) : La Construction de la réalité sociale, Paris, Gallimard.

SPERBER, D. (1996) : La Contagion des idées, Paris, Odile Jacob.

STRAWSON, P. ([1959] trad. fr. 1973) : Les Individus, Paris, Les Éditions du Seuil.

SUMPF, J. (1984) : « Noms - Noms propres », Langages 76, 113-128.

VignauX, G. (1999) : Le Démon du classement. Penser, organiser, Paris, Les Éditions du Seuil.

V OLTAIRE ([1764] rééd. 1987) : Dictionnaire philosophique, R. Naves \& J. Benda (éds.), Paris, Garnier, coll. Classiques.

WiERZBICKA, A. (1988) : The Semantics of Grammar, Amsterdam-Philadelphia, Benjamins.

- (1996) : Semantics : Primes and Universals. Oxford University Press

Wiggins, D. (1980) : Sameness and Substance, Oxford, Blackwell.

WittGenstein, L. ([1922] trad. et éd. fr. 1993) : Tractatus logico-philosophicus, G.-G. Granger (éd.), Paris, Gallimard.

ZONABEND, F. (1977) : «Pourquoi nommer?», repris in Claude Lévi-Strauss (dir.), 1987, L'Identité, Paris.

\section{Corpus}

A PULÉE, $2^{\text {e }}$ siècle après J.-C., L'Âne d'or ou les métamorphoses, trad. fr. P. Grimal 1958, rééd. Paris, Gallimard-Folio, 1975.

Belletto, R. (1990) : La Machine, rééd. 1991, Paris, J'ai lu.

ERCKMANN (Émile) - CHATRIAN (Alexandre) (1859) : Les trois âmes, in Contes fantastiques complets, rééd. Paris, Néo, 1987.

GAUTIER, T. (1856) : Avatar, in La Morte amoureuse et autres récits fantastiques, J. Gaudon (éd.), Paris, Gallimard, Coll. Folio, 1981.

O’BRIEN, F. J. (1858) : La Chambre perdue, trad. fr. J. Papy, in J. Goimard \& R. Stragliati (éds), 1996, La Grande Anthologie du fantastique 1, Paris, Omnibus, 772-790.

Mallarmé, S. (1893) : Contes indiens, in Euvres complètes, éd. B. Marchal, Paris, Gallimard, coll. La Pléiade, 1998.

STEVEnson, R. L. (1886) : L'Étrange Cas du Dr Jekyll et de Mr Hyde, trad. J.P. Naugrette, Paris, Librairie Générale Française, LGF, le Livre de Poche bilingue, 1988.

Wells, H. G. (1896) : L'Île du Docteur Moreau, trad. fr. H.-D. Davray, Paris, Gallimard, coll. folio, 1975. 